\title{
MANAGEMENT OF DISPLACED TIBIAL SPINE FRACTURE - A PROSPECTIVE STUDY
}

Dibakar Ray, Prasanta Kumar Mandal, Fagu Ram Maji, Somnath Tirkey, Mrinal Kanti Ray, Amalendubikas Chatterjee

1. Assistant Professor, Department of Orthopaedics, Bankura Sammilani Medical College \& Hospital, Bankura.

2. Associate Professor, Department of Orthopaedics, Bankura Sammilani Medical College \& Hospital, Bankura.

3. Assistant Professor, Department of Orthopaedics, Bankura Sammilani Medical College \& Hospital, Bankura.

4. Junior Resident, Bankura Sammilani Medical College \& Hospital, Bankura.

5. Assistant Professor, Department of Orthopaedics, Bankura Sammilani Medical College \& Hospital, Bankura.

6. Associate Professor, Department of Anaesthesia, Bankura Sammilani Medical College \& Hospital, Bankura.

\section{CORRESPONDING AUTHOR}

Dr. Dibakar Ray,

C/O, Kamala Laya, Ramkrishna Pally,

Benachity, Durgapur-713213.

E-mail: dibakar74@yahoo.com

Ph: 009198030350258

ABSTRACT: Anterior tibial spine fracture is relatively a rare injury in orthopedic practice. Undisplaced and minimally displaced (type 1\&2) fractures can be treated by closed reduction and long leg cast. To avoid persistent post injury instability and secondary degenerative changes displaced tibial spine fractures (type3) need to be managed surgically. In this study, all displaced tibial spine fractures (type3) are treated with open reduction and internal fixation with stainless steel wires except in one case which is fixed with non absorbable suture. During follow up very good result are obtained in respect to satisfactory reduction, healing of fractures, and range of motion and stability of knee.

KEY WORDS displaced tibial spine, stainless steel wire fixation

INTRODUCTION - As reported in literature avulsion fractures of tibial spine is relatively rare injury in children \& adolescents*(3 per 10000 per year and minimally displaced fractures can be managed conservatively but to avoid persistent post injury instability and secondary degenerative changes completely displaced tibial spine fracture should be treated by open reduction and internal fixation followed by proper post operative rehabilitation programme. In this series 15 such consecutive displaced tibial spine fractures were selected for operative treatment and their results were evaluated.

MATERIALS \& METHODS: 15 patients aged 15 to 30 years with displaced fractures were treated from June 2011 to July 2012. Male female ratio of the patents was 7:3.most of them was either due to athletic activity or fall from bicycle. Patient received treatments earliest on $6^{\text {th }}$ day and latest 6 month after injury. Of 15,9 patient were treated 6 day after injury and 3 cases were delayed for 6 months. Others were treated between 12 -30 days after injury. Classification system of Meyers and Mckeever was used to describe the degrees of displacement of tibial spine fractures .All cases who had type 3 fracture were treated by open reduction and internal fixation. None of them were associated with bony or collateral ligamentous injuries on examination. Among 12 type 3 fracture were fixed by $20 \mathrm{~g}$ stainless steel wire; and polypropylene (prolene) was used in one cases only. 
All patients were examined thoroughly to rule out any associated medial collateral ligament, lateral collateral ligament, physeal fracture of distal femur, proximal tibia and meniscal injuries. All patients had undergone radiographic evaluation to assess the degree of displacement.

Operative procedure all type 3 fractures were approached through antero medial arthrotomy .After opening the capsule medially, the medial meniscus was examined. Retraction was used to allow the examination of anterior horn of lateral meniscus. In one 16 year old child the fragment was reduced in tibial epiphysiseal crater and secured with nonabsorbable suture (Prolene).In other patients, from an anteromedial starting point on the tibial metaphysis, drill holes were placed obliquely into the crater in the tibial epiphyses. The fragment was reduced in its bed and wire was passed through the drill holes anteromedially with suture passer keeping the knee in extension and tied securely. In 3 old cases, fracture fragments were freshened from all fibrous tissue and crater was curetted for adequate reduction.

Post-operative management after reduction and fixation a long leg plaster cast was applied with knee in full extension. The cast was kept for 6 week .Strengthening exercise of the quadriceps and the hamstring were started immediately. Gradual weight bearing was allowed from toe touchdown to full weight bearing subsequently.

RESULTS: The results were evaluated in terms of range of motion, subjective instability and pain. Painless near full range of motion (I5 to135 degree)without having subjective instability was marked as excellent( 5 cases).loss of extension ( 10 degree)without subjective instability and pain was marked as good ( 7 cases)and 30degree or more loss of extension and mild pain during walking( 3 cases) was marked as fair.

DISCUSSION: In all 15 patients we did not find any associated ligamentous injury .Though Garcia \& Neer reported 42 fractures of the tibial spine in patients aged 7 to 60 years. Six of them were positive anterior drawer sign indicating associated or cruciate ligament injuries.

The procedure followed in this series was same as recommended by Roberts and Lovell (most anterior tibial spine should be treated by closed reduction \&immobilization in extension and open reduction \&internal fixation for irreducible fractures)

No subjective complaints of knee instability was found during follow up, but objective evidence of anterior instability was found in 33\%of cases .According to Gronkvist et al . Younger children will compensate somewhat for any anterior instability as the skeleton grows but in older children some anterior instability may persist, that can be explained by the study of Willis et,al. who noted that most children have objective evidence of ACL laxity at long term follow up but few have subjective complaints.

In $80 \%$ patient we have achieved excellent to good results as most patients regained an excellent range of motion with loss of extension ranging from 5-15 degree but 3 cases (20\%)did not achieved their terminal 30 degree of extension probably due to malunion and delayed presentation.We removed all hardwires after 6 months of operation.

CONCLUSION: Though avulsion fractures of tibial spine is a rare injury, the diagnosis can be made with a high degree of clinical suspicion and good quality of roentgenogram. It is commonly seen in children and adolescent males. Displaced fractures need to be treated by arthrotomy and open reduction and internal fixation followed by cast immobilization with knee 
in extension. Patients should be treated early for good results, keeping the knee instability and knee laxity in mind.

\section{REFERENCES:}

1. Bakalim G, Wilpulla C : Closed redction of fracture of the tibial spines. INJURY 1974;5:210-212

2. Baxter MP,Wiley JJ.Fractures of thetibial spine in children: an evaluation of knee instability.J BoneJoint SurgeryBr 1988;70 228-230

3. Canale ST.Fractures and dislocations. In: Canale ST, Beaty JH, eds Operative Pediatric Orthopedics.

4. Garcia A, NeerCSII.Isolated fractures of intercondylar eminence of the tibia.Am J Surg 1958; 95:593-598

5. Gronkvist H, Hirsch G, Johansson L. fractures of the ant tibial spine in children. J Pediatric Orthop 1984:4:465-468

6. Janarv P-M, Westblad P, Johansson $\mathrm{C}$, et al.Long term follow up of ant tibial spine fractures in children J Ped Orthop 1995; 15:63-68.

7. Meyers MH, Mckeever FM Fractures of the intercondylar eminence of yhe tibia.J B J S Am 1959; 41:209-222

8. Pellaci F, Mignani G, Valdiserri L.Fractures of the intercondylar eminence of the tibia in children. Ital JOrthop traumatol 1986; 12: 441-446.

9. Roberts JM, Lovell WW Fractures of the intercondylar eminence of the tibia . JBJS Am 1970;52:827.

10. Willis RB , Blokker C,Stoll TM, et al . J Ped Orthop 13;361,1993.

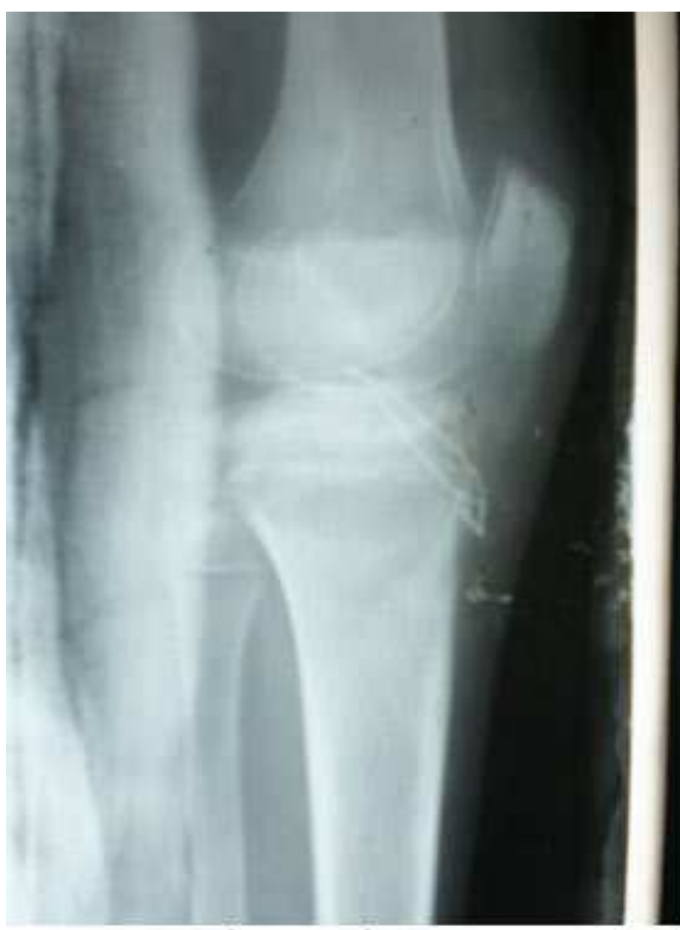

post op fg1 patient lat view

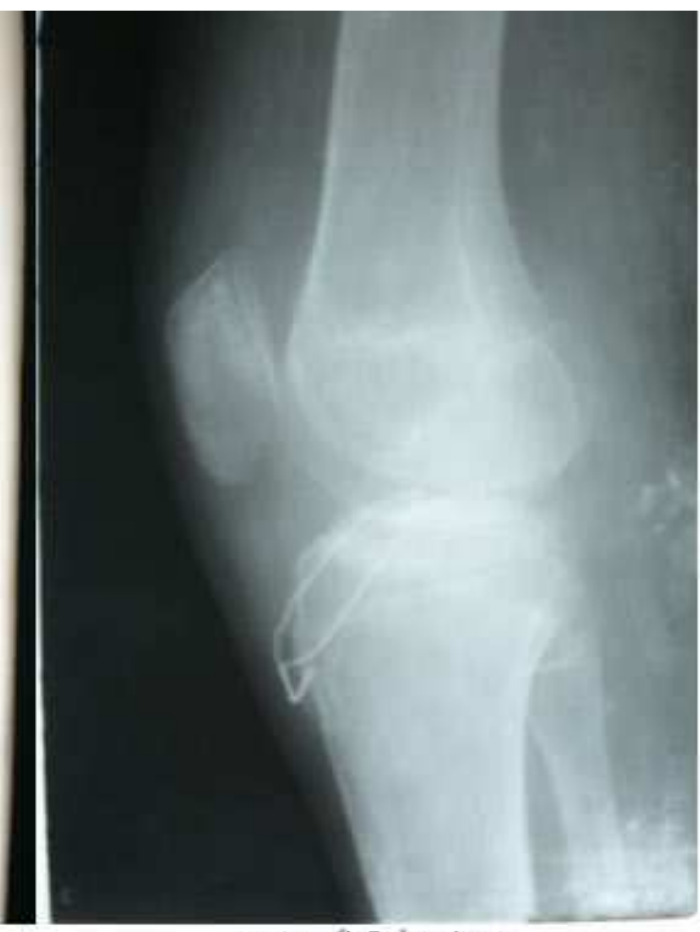

post op fig2 -lat view 


\section{ORIGINAL ARTICLE}

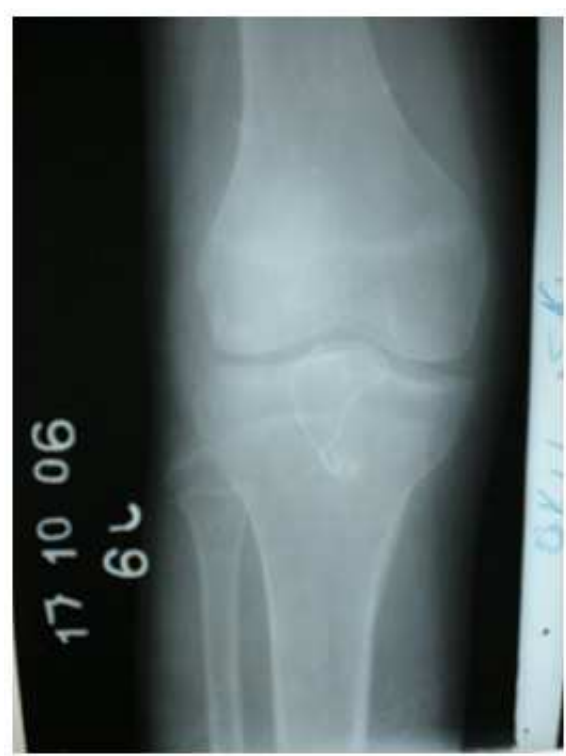

post op fig2 patient A.P view

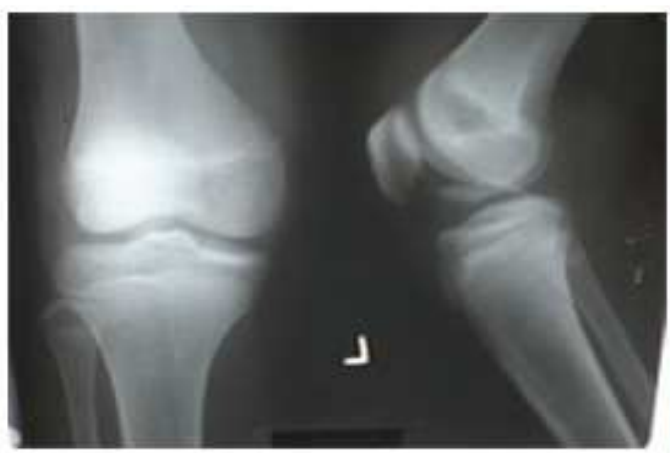

pre op fig1 showing displaced tibial spine fracture

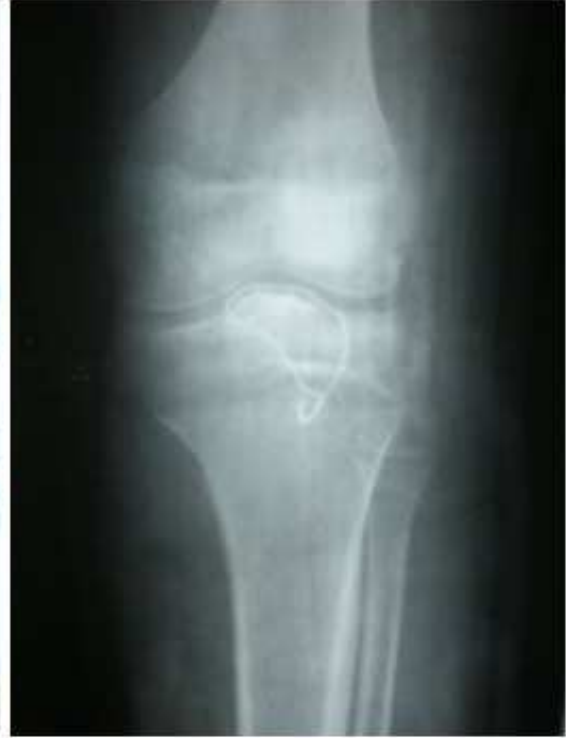

post op same fg1 patient A.P view

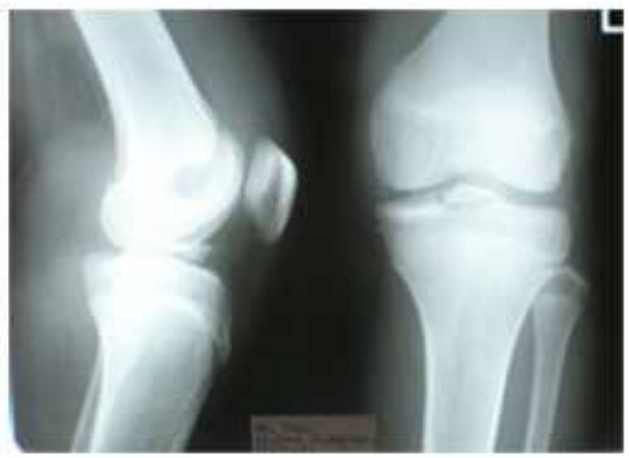

pre op fig 2 patient showing displaced tibial spine fracture

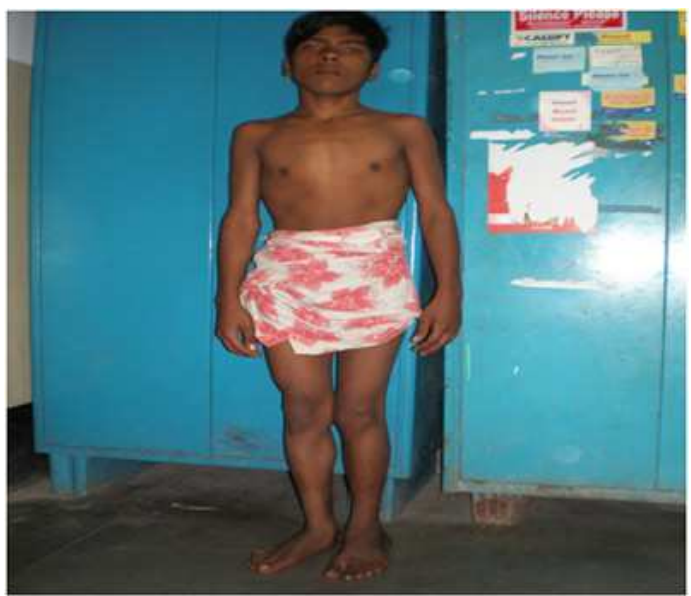

Post op follow up of the patient 